\title{
Factors Associated with Hospital-Acquired Delirium in Patients 18-65 Years Old
}

J Gen Intern Med 36(4):1147-9

DOI: $10.1007 / \mathrm{s} 11606-020-06378-\mathrm{w}$

(C) Society of General Internal Medicine 2021

\section{INTRODUCTION}

Delirium may affect hospitalized patients of any age, but delirium initiatives have focused mostly on the elderly as older patients are more predisposed to developing delirium. Delirium has been reported in 11-42\% of the hospitalized patients over the age of 65 years. ${ }^{1}$ The few studies that reported delirium in patients between the ages of 18 and 65 years were small and focused on oncology or palliative care. In these populations, delirium was reported in $30-50 \%$ of the patients..$^{2-4}$

In this study, we assess the rate of delirium among hospitalized adults between the ages of 18 and 65 years, and the factors associated with delirium among these patients.

\section{METHODS}

This is a retrospective study comprising 95,304 discharges between November 1, 2013, and January 31, 2018, at our institution. Patients were included if they were 18-65 years of age and did not have a diagnosis of delirium on admission. Patients with a length of stay (LOS) of less than $24 \mathrm{~h}$ were excluded. Delirium was identified using International Classification of Diseases codes (Table 1). This study was determined to be exempt from review by our Institutional Review Board.

We first conducted a bivariate analysis to identify the factors associated with delirium. For each variable, we calculated the standardized mean difference (SMD) between patients without and with delirium. Variables with $\mathrm{SMD} \geq 0.20$ were included in the multivariate logistic regression analysis to determine which variables were independently associated with delirium. All analyses were done using R 3.4.3 base.

\section{RESULTS}

For patients aged 18-65 years, among 95,304 discharges, 2855 (3.0\%) were diagnosed with delirium (compared to $5.5 \%$ of the 59,940 discharges among patients aged $>65$ years).

Received March 10, 2020

Accepted December 1, 2020

Published online January 8, 2021
Delirium in patients aged 18-65 years thus accounted for $48.1 \%$ of all the cases with delirium among hospitalized adults.

Table 1 compares the characteristics of patients with and without delirium. Compared to patients without delirium, patients with delirium had a longer median LOS (12.1 versus 3.0 days), higher inpatient mortality ( $13.2 \%$ versus $0.9 \%)$, and more discharges to nursing home $(17.5 \%$ versus $4.2 \%)$. Among patients with delirium, the most common discharging services were neurosurgery $(9.0 \%)$, general medicine $(8.0 \%)$, general surgery $(7.2 \%)$, hematology/oncology $(5.6 \%)$, and orthopedic surgery $(3.8 \%)$.

Independent predictors of delirium were age, Charlson comorbidity score, intensive care unit (ICU) stay, and administration of benzodiazepines, anticholinergics, or corticosteroids (Table 2). Opiate use was not associated with delirium. The $c$-statistic of this model was 0.83 .

\section{DISCUSSION}

The variables most strongly associated with delirium among patients 18-65 years old were ICU stay (especially $>3$ days) and administration of benzodiazepines. This is similar to the factors associated with delirium in older patients. ${ }^{5}$ While ICU stay was a strong predictor of delirium in our study, $42.3 \%$ of the patients with delirium did not have an ICU stay. Additional factors associated with delirium were older age, more comorbidities, and use of anticholinergics and corticosteroids. Patients with delirium had longer LOS, higher mortality, and more discharges to nursing home, akin to patients $>65$ years old. ${ }^{6}$

\section{Limitations}

First, this is a single-center study and the results may not be generalizable. We did, however, have a large database, including 95,304 discharges, which allowed us to assess the variables that were independently associated with delirium. Second, we used ICD codes to identify delirium, which is limited by the quality of physician documentation. This may explain the relatively low rate of delirium in our study compared to smaller studies that reported higher rates based on prospective screening or extensive chart review in specific high-risk populations. ${ }^{2-4}$ Third, our database did not differentiate home medications from new hospital prescriptions, or if these medications were given before or after the onset of delirium. We, therefore, cannot exclude the possibility that the association between benzodiazepine use and delirium could be a result of 
Table 1 Characteristics of Patients 18-65 Years of Age, Without and With Delirium

\begin{tabular}{|c|c|c|c|}
\hline & $\begin{array}{l}\text { Without delirium } \\
(n=92,449)\end{array}$ & $\begin{array}{l}\text { With delirium }^{\mathrm{a}} \\
(n=2855)\end{array}$ & $\begin{array}{l}\text { Standardized mean } \\
\text { difference }^{\mathrm{d}}\end{array}$ \\
\hline Age at admission, median (IQR), $y$ & $50(38,58)$ & $54(42,60)$ & 0.21 \\
\hline Male, no. $(\%)^{\text {b }}$ & $44,669(48.3)$ & $1653(57.9)$ & 0.19 \\
\hline Non-Hispanic, no. $(\%)^{\mathrm{b}}$ & $71,955(77.8)$ & $2207(77.3)$ & 0.07 \\
\hline Married/partnered, no. $(\%)^{\mathrm{b}}$ & $50,380(54.5)$ & $1497(52.4)$ & 0.09 \\
\hline Case mix index, median $(\mathrm{IQR})^{\mathrm{b}}$ & $1.6(1.0,2.3)$ & $4.7(1.9,7.9)$ & 0.91 \\
\hline Case mix index $>2.5$, no. $(\%)^{\mathrm{b}}$ & $15,826(21.6)$ & $1683(67.6)$ & 1.04 \\
\hline \multicolumn{3}{|l|}{ Charlson comorbidity score, no. (\%) } & 0.49 \\
\hline 0 & $35,170(38.0)$ & $625(21.9)$ & \\
\hline $1-2$ & $32,669(35.3)$ & $826(28.9)$ & \\
\hline $3-4$ & $12,111(13.1)$ & $672(23.5)$ & \\
\hline $5+$ & $12,499(13.5)$ & $732(25.6)$ & \\
\hline \multicolumn{3}{|l|}{ Discharging service, no. $(\%)$} & 0.08 \\
\hline Surgical & $42,635(46.2)$ & $1209(42.4)$ & \\
\hline Medical & $49,814(53.8)$ & $1646(57.6)$ & \\
\hline \multicolumn{3}{|l|}{ ICU stay, no. (\%) } & 1.20 \\
\hline Never ICU & $81,900(88.6)$ & $1207(42.3)$ & \\
\hline $1-3$ days & $4806(5.2)$ & $167(5.8)$ & \\
\hline$>3$ days & $5743(6.2)$ & $1481(51.9)$ & \\
\hline \multicolumn{4}{|l|}{ Medications administered, no. $(\%)^{\mathrm{c}}$} \\
\hline Opiates & $70,416(76.2)$ & $2443(85.6)$ & 0.24 \\
\hline Benzodiazepines & $24,425(26.4)$ & $1580(55.3)$ & 0.62 \\
\hline Anticholinergics & $28,268(30.6)$ & $1353(47.4)$ & 0.35 \\
\hline Corticosteroids & $21,197(22.9)$ & $1217(42.6)$ & 0.43 \\
\hline Antipsychotics & $7049(7.6)$ & $1595(55.9)$ & 1.21 \\
\hline Zolpidem & $7004(7.6)$ & $346(12.1)$ & 0.15 \\
\hline Trazodone & $6064(6.6)$ & 645 (22.6) & 0.47 \\
\hline Alcohol/drug use, no. (\%) & $1113(1.2)$ & $110(3.9)$ & 0.17 \\
\hline Length of stay, median (IQR), $d$ & $3.0(1.6,5.2)$ & $12.1(5.8,24.6)$ & 0.84 \\
\hline Inpatient mortality, no. (\%) & $808(0.9)$ & $376(13.2)$ & 0.49 \\
\hline Discharge to nursing home, no. (\%) & $3910(4.2)$ & $499(17.5)$ & 0.44 \\
\hline
\end{tabular}

$I Q R$, interquartile range; $I C U$, intensive care unit

${ }^{a}$ Delirium was identified using International Classification of Disease 9 codes (clinical modification): 293.0, 780.79, 780.1, 780.97, 307.9, 348.3, 348.30,

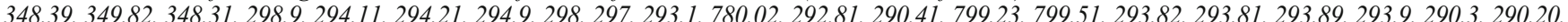
290.11, 290.12. Delirium was also identified using International Classification of Disease 10 codes: F05, F05.0, F05.1, F05.8, F05.9, R41.0, F06.0, F06.2, F06.8, F09, F11.121, F11.122, F11.150, F11.151, F11.159, F11.221, F11.222, F11.129, F11.229, F11.23, F11.250, F11.251, F11.259, F11.920, F11.921, F11.922, F11.950, F11.951, F11.959, F11.929, F11.93, F13.239, F13.230, F13.121, F13.150, F13.151, F13.159, F13.231, F13.232, F13.250, F13.251, F13.259, F13.921, F13.931, F13.932, F13.950, F13.951, F13.959, F13.939, F13.930, F13.929, F13.920, F13.129, F13.120, F29, F22, F23, F24, R40.0, R41.0, R41.82, R41.89, R41.9, G93.4, G93.40, G93.49, G93.41, R40.4, R44, R44.0, R44.1, R44.2, R44.3, R44.8, R44.9, R45.1, R45.6, R45.87, R41.840. 3431 patients who had a diagnosis of delirium present on admission were not included

${ }^{b}$ Missing values: Sex -2 encounters without delirium and 0 encounters with delirium; Ethnicity - 1410 encounters without delirium and 72 encounters with delirium; Marital status - 493 encounters without delirium and 39 encounters with delirium; Case mix index - 19,099 encounters without delirium and 364 encounters with delirium

${ }^{c}$ If patient received any dose of this class of medications during that hospital encounter, it was counted. Anticholinergics included were based on our hospital formulary: amitriptyline, clomipramine, desipramine, diphenhydramine, hydroxyzine, imipramine, nortriptyline, oxybutynin, scopolamine, tolterodine, cyclobenzaprine, promethazine, and prochlorperazine

${ }^{d}$ Standardized mean difference (SMD) was calculated using the method implemented in the "tableone" package in $R 3.4 .3$ base. In our analysis, SMD $\geq$ 0.20 was interpreted as a meaningful difference and shown in bold text in the table. SMD is a measure of effect size and defined as the mean difference of a variable between the two groups divided by the pooled standard deviation. SMD adjusts for the sample size. An SMD of 0 means there was no observed difference between patients with and without delirium for that variable. SMD greater than 0 indicates the degree of difference between the two groups for that variable. The higher the SMD, the greater the magnitude of association in the bivariate analysis

clinicians treating delirium with benzodiazepines. This, however, does not affect our other findings, as excluding benzodiazepine use from our multivariate model did not alter the associations of the other predictor variables with delirium.

In conclusion, we demonstrated that among hospitalized patients aged 18-65 years, patients at highest risk of delirium were those who had an ICU stay and those who received benzodiazepines. These at-risk patients should therefore be considered for inclusion in delirium screening and prevention initiatives throughout their hospital stay.

Nidhi Rohatgi, $M D, M S^{1,2}$

Yingjie Weng, $M H S^{3,2}$

Neera Ahuja, $M D^{1,2}$

Maarten G. Lansberg, $M D, P h D^{4,2}$
${ }^{1}$ Division of Hospital Medicine, Department of Medicine, Stanford University School of Medicine, Stanford, CA, USA

${ }^{2}$ Stanford, USA

${ }^{3}$ Quantitative Sciences Unit, Division of Biomedical Informatics Research, Department of Medicine, Stanford University School of Medicine,

Stanford, CA, USA

${ }^{4}$ Department of Neurology and Neurological Sciences, Stanford University School of Medicine,

Stanford, CA, USA

Corresponding Author: Nidhi Rohatgi, MD, MS; Stanford, USA (e-mail: nrohatgi@stanford.edu). 
Table 2 Multivariate Analysis to Identify Factors Associated with Delirium During the Hospital Stay for Patients 18-65 Years of Age

\begin{tabular}{|c|c|c|c|}
\hline Variables $^{\mathrm{a}}$ & $\begin{array}{l}\text { Odds } \\
\text { ratio }\end{array}$ & $\begin{array}{l}95 \% \text { confidence } \\
\text { interval }\end{array}$ & $P$ value \\
\hline Age, per 10 years & 1.14 & 1.10 to 1.18 & $\begin{array}{l}< \\
0.0001\end{array}$ \\
\hline \multicolumn{4}{|c|}{ Charlson comorbidity score } \\
\hline 0 (reference) & - & - & - \\
\hline $1-2$ & 0.90 & 0.81 to 1.00 & 0.05 \\
\hline $3-4$ & 1.33 & 1.17 to 1.50 & $\begin{array}{l}< \\
0.0001\end{array}$ \\
\hline $5+$ & 1.54 & 1.37 to 1.74 & $\begin{array}{l}< \\
0.0001\end{array}$ \\
\hline \multicolumn{4}{|l|}{ ICU stay, $d$} \\
\hline $\begin{array}{l}\text { Never ICU } \\
\text { (Reference) }\end{array}$ & - & - & - \\
\hline $1-3$ & 3.63 & 3.24 to 4.07 & $\begin{array}{l}< \\
0.0001\end{array}$ \\
\hline$>3$ & 22.36 & 20.12 to 24.84 & $\begin{array}{l}< \\
0.0001\end{array}$ \\
\hline \multicolumn{4}{|c|}{ Medications administered $^{\mathrm{b}}$} \\
\hline Opiates & 0.93 & 0.83 to 1.04 & 0.17 \\
\hline Benzodiazepines $^{\mathrm{c}}$ & 2.52 & 2.32 to 2.74 & $\begin{array}{l}< \\
0.0001\end{array}$ \\
\hline Anticholinergics & 1.40 & 1.28 to 1.52 & $\begin{array}{l}< \\
0.0001\end{array}$ \\
\hline Corticosteroids & 1.27 & 1.16 to 1.38 & $\begin{array}{l}< \\
0.0001\end{array}$ \\
\hline
\end{tabular}

ICU, intensive care unit

${ }^{a}$ Demographic and clinical variables with standardized mean difference $(S M D) \geq 0.20$ in the bivariate analysis were included into a multivariate model to determine which variables are independently associated with delirium. The following variables were included in the multivariate model: age, Charlson comorbidity score, ICU stay, opiates, benzodiazepines, anticholinergics, and corticosteroids. The following variables were not included in the multivariate model despite $S M D \geq 0.20$ : (a) Case mix index (there were multiple missing values: 19,099 encounters with missing values in the group without delirium and 364 encounters with missing values in the group with delirium), and (b) antipsychotics and trazodone (used in management of delirium)

${ }^{b}$ If patient received any dose of this class of medications during that hospital encounter, it was counted. Anticholinergics included were based on our hospital formulary: amitriptyline, clomipramine, desipramine, diphenhydramine, hydroxyzine, imipramine, nortriptyline, oxybutynin, scopolamine, tolterodine, cyclobenzaprine, promethazine, and prochlorperazine

${ }^{c}$ Administration of benzodiazepine was found to be one of the strongest predictors of incident delirium in the model. Our dataset does not allow differentiating home medications from new prescriptions in the hospital or if the medications were started before or after the onset of delirium. Since benzodiazepines could sometimes be used for treatment of delirium, we ran this multivariate analysis without including benzodiazepine use as a predictor variable, and the results remained largely unchanged

\section{Compliance with Ethical Standards:}

Conflict of Interest: The authors declare that they do not have a conflict of interest.

\section{REFERENCES}

1. Siddiqi N, House AO, Holmes JD. Occurrence and outcome of delirium in medical in-patients: a systematic literature review. Age Ageing. 2006;35(4):350-364. https://doi.org/10.1093/ageing/afl005.

2. Weckmann MT, Bay C, Abu Ata N, Morrison RS. Incidence and Cause of Delirium in Hospitalized Patients between the Ages of 18 and 56: A Retrospective Chart Review. Int J Palliat Care. 2014;2014: 1-7. https://doi. org/10.1155/2014/214265.

3. Elsayem AF, Bruera E, Valentine AD, et al. Delirium frequency among advanced cancer patients presenting to an emergency department: A prospective, randomized, observational study. Cancer. 2016;122(18):29182924. https://doi.org/10.1002/cncr.30133.

4. Fann JR, Hubbard RA, Alfano CM, Roth-Roemer S, Katon WJ, Syrjala KL. Pre- and post-transplantation risk factors for delirium onset and severity in patients undergoing hematopoietic stem-cell transplantation. J Clin Oncol. 2011;29(7):895-901. https://doi.org/10.1200/JCO.2010.28. 4521.

5. Ahmed S, Leurent B, Sampson EL. Risk factors for incident delirium among older people in acute hospital medical units: a systematic review and meta-analysis. Age Ageing. 2014;43(3):326-333. https://doi.org/10. 1093/ageing/afu022.

6. Schubert $\mathbf{M}, \mathbf{S c h u ̈ r c h} \mathbf{R}$, Boettger $\mathbf{S}$, et al. A hospital-wide evaluation of delirium prevalence and outcomes in acute care patients - a cohort study. BMC Health Serv Res. 2018;18(1):550. https://doi.org/10.1186/s12913018-3345-x.

Publisher's Note: Springer Nature remains neutral with regard to jurisdictional claims in published maps and institutional affiliations. 